\title{
4. PREJUICIOS ENTRE LOS ESTUDIANTES HACIA LAS PERSONAS CON DISCAPACIDAD: REFLEXIONES A PARTIR DEL CASO DE LA UNIVERSIDAD DE ALICANTE*
}

\author{
Student prejudices towards people with disabilities: \\ reflections based on the case of the University of Alicante
}

\author{
RAQUel SURIÁ MARTíneZ** \\ Agustín Bueno Bueno*** \\ Ana María Rosser LimiñanA****
}

\section{Resumen}

Introducción. Evaluar la actitud que los estudiantes muestran hacia la discapacidad es fundamental para la integración de estos alumnos; por ello, existe en nuestro país una gran cantidad de investigaciones en esta materia, aunque son escasas las centradas en estudiantes universitarios.

Recibido: $28 / 10 / 2010$

Revisado: 01/12/2010

Aceptado: 07/02/2011

** Raquel Suriá Martínez

Responsable correspondencia.

Departamento de Comunicación y Psicología Social. Grupo de Innovación Tecnológica Educativa de Psicología Social (GPSOC). Universidad de Alicante.

Despacho 0034PB016

Carretera San Vicente del Raspeig s/n

03690 San Vicente del Raspeig - Alicante

Tel.: 965903400 (ext. 2182)

raquel.suria@ua.es

*** Agustín Bueno Bueno

Departamento de Comunicación y Psicología Social. Grupo de Innovación Tecnológica Educativa de Psicología Social (GPSOC). Universidad de Alicante.

**** Ana María Rosser Limiñana

Departamento de Comunicación y Psicología Social. Grupo de Innovación Tecnológica Educativa de Psicología Social (GPSOC). Universidad de Alicante. 
Objetivos. El propósito del estudio consiste en evaluar las actitudes de los estudiantes universitarios hacia los alumnos discapacitados en función del curso y la titulación de los estudiantes.

Método. Participaron 340 alumnos de la Universidad de Alicante. Diseñamos un cuestionario donde se estiman las actitudes de los estudiantes.

Resultados. Los resultados indican diferencias en las actitudes de los estudiantes en función del curso y de la titulación.

Discusión y conclusiones. Nuestro estudio muestra que, en general, los alumnos presentan actitudes favorables hacia los alumnos con discapacidad, no obstante, encontramos que desde ciertas titulaciones, y en los cursos superiores, los estudiantes indican mayor nivel de sensibilización hacia la discapacidad.

Palabras clave: discapacidad, estudiantes, universidad, prejuicios, integración.

\begin{abstract}
Introduction. The need to assess the attitudes that students show towards disability is essential for the integration of students with disabilities. In our country there is a lot of research on this subject, but none focused on university students.

Objectives. Our study examines students' attitudes toward students with disabilities depending on their course year and degree.

Method. 340 students at the University of Alicante participated. We designed a questionnaire that estimates the attitudes of students.

Results. The results indicate differences in the attitudes of participating students on the basis of the course year and the degree.

Discussion or Conclusion. Our study shows that the students show positive attitudes towards students with disabilities however, we found that in some degrees and in the higher course years, students indicated a higher level of awareness of disability.
\end{abstract}

Keywords: disabilities; students; university; prejudices; integration.

\title{
1. Introducción
}

Desde hace algunos años, el interés desde diferentes ámbitos por atender el mundo de la discapacidad, al igual que la proliferación de investigaciones dedicadas a la integración de este colectivo, se ha incrementado notablemente.

Uno de los campos más implicados en esta temática es el ámbito educativo, en el que el tratamiento que se da a la diversidad en nuestro país facilita la integración de estudiantes que conviven día a día con esta problemática, trasladándose esto a un aumento del número de alumnos que no solo acceden a la formación primaria y secundaria sino a cursar estudios superiores (Castellana y Sala, 2005; Eches y Ochoa, 2005; Konur, 2006).

Sin embargo, aunque es cierto que existe un número creciente de jóvenes con discapacidad que desean formar parte del mundo universitario (Konur, 2006), 
todavía son numerosas las ocasiones en las que encontramos que la universidad es una de las instituciones más excluyentes para el ingreso y permanencia de estos estudiantes (Cuskelly y Bryde, 1994; Moreno, Rodríguez, Saldaña y Aguilera, 2006).

En este sentido, son muchos los factores que se deben tener en cuenta desde cualquier política educativa, que van desde la supresión de barreras arquitectónicas hasta las adaptaciones curriculares. Sin embargo, una de las variables más influyentes que pueden afectar a los estudiantes con discapacidad, y que tienen un caracter invisible, se refiere a las barreras mentales de los miembros de la universidad.

Con respecto a esta variable, la literatura existente incide en que el éxito de un sistema educativo integrador está directamente relacionado con las actitudes positivas de la comunidad educativa (Bunch y Valeo, 2004; Burstein, Sears, Wilcoxen, Cabello y Spagna, 2004; Estévez, Murgui, Moreno y Musitu, 2007; Garaigordobil y García de Galdeano, 2006; Navas, Torregrosa y Mula, 2004), siendo las actitudes del resto de compañeros de los estudiantes con discapacidad uno de los elementos clave para la plena integración de este colectivo. Pero ¿qué sabemos de las actitudes que tienen los estudiantes que pasan parte de la vida académica con alumnos con discapacidad?, ¿son positivas?, ¿pueden variar?, ¿qué variables del ámbito universitario pueden influir en dichas actitudes?

Con relación a estos aspectos, si la actitud de los estudiantes no discapacitados ante los alumnos con discapacidad es un asunto con consecuencias importantes en la integración social de las personas con algún tipo de discapacidad, lo es más cuando los alumnos no discapacitados son, precisamente, aquellos estudiantes que eligen titulaciones que, en un futuro, están dirigidas a la rehabilitación de estas personas, por su integración social, educativa y laboral y, en definitiva, porque se vean satisfechas sus necesidades especiales (Nowicki, 2006; Verdugo, Jenaro y Arias, 1995).

Ante esto, ¿qué sabemos sobre las actitudes de los estudiantes que se están formando en titulaciones relacionadas con este ámbito de actuación?; los alumnos que eligen estas carreras, ¿tienen una actitud más positiva que los que eligen otro tipo de carreras?; si estas actitudes son más positivas, ¿parten al inicio de su carrera con esta actitud? o por el contrario, ¿van evolucionando según avanzan en su etapa formativa?

Siguiendo las investigaciones que hacen referencia a las titulaciones y su relación con las actitudes que tienen los universitarios sobre los estudiantes con discapacidad, nuestros estudios se han centrado en titulaciones específicas (Alpuín, González y Pérez, 2006) y en materias que guardan relación con la discapacidad (Moreno et al. 2006). Por tanto, resulta difícil concluir si los resultados sobre las actitudes hacia el alumnado discapacitado vienen determinadas por la titulación analizada o por otras variables relacionadas con los alumnos. 
Para examinar esta posible hipótesis, algunas investigaciones se han centrado en otras variables inherentes a los estudiantes como, por ejemplo, la edad y el género. En este sentido, los estudios sobre actitudes en función de la edad se ha centrado preferentemente en la evaluación de las actitudes de estudiantes de primaria hacia sus iguales con discapacidad (Aguado, Flórez y Alcedo, 2004; Avramidis y Norwich, 2000; Van-Reusen, Shoho y Barker, 2000; Verdugo, Arias y Jenaro, 1994), desconociendo si las actitudes varían en función de la edad en otras etapas formativas.

Por su parte, el género de los compañeros de los alumnos con discapacidad es otra de las variables estudiadas en las actitudes hacia la discapacidad, aunque los resultados sobre este factor son contradictorios (Aguado, Flórez y Alcedo, 2004; Nowicki, 2006; Wolpe, 1997).

No obstante, aunque los estudios no revelen directamente diferencias en el género y actitud, podríamos plantearnos su influencia de manera indirecta, es decir, ¿podría esta variable reflejar su influencia en las actitudes hacia la discapacidad a través de otras variables implicadas, como la titulación que uno estudie?

En este sentido, como indica la Comisión Europea en el informe elaborado por la Red de Expertos en Ciencias Sociales de la Educación (NESSE, 2009) sobre la dimensión hombres-mujeres y la educación, se observan las diferentes elecciones según las ramas de estudios, encontrándonos por tanto con ramas claramente feminizadas o masculinizadas (Lynch y Feeley, 2009). Por ejemplo, las mujeres continúan siendo la gran mayoría en las especialidades consideradas tradicionalmente femeninas (Humanidades, Artes, Enfermería, Servicios Sociales, etc.) y una minoría en las carreras consideradas masculinas (Ingeniería, Arquitectura, Informática, etc.).

En este contexto cobra relevancia nuestro estudio puesto que, examinando las actitudes hacia los estudiantes con discapacidad que tienen los alumnos procedentes de diversas titulaciones y de diferentes cursos, se puede detectar si alguna de estas variables incide en las actitudes y, de este modo, llevar a cabo una intervención para modificarlas o mejorarlas.

Así, los objetivos del estudio consisten en:

1. Identificar si existen diferencias entre las actitudes de los estudiantes hacia los alumnos con discapacidad en función de la titulación en la que se encuentran los alumnos participantes del estudio.

1.1. En este sentido, esperamos que los alumnos de titulaciones que guardan relación con la atención a la diversidad y a la discapacidad tengan una actitud más positiva hacia el alumnado con discapacidad.

1.2. Con relación a esto hemos mencionado la posible relación entre la titulación y el género de los participantes, por lo que nuestro segundo objetivo consiste en examinar si el género influiría en la 
titulación cursada por los estudiantes, y si este es el caso, averiguar en qué dirección lo hace.

2. Examinar si existen diferencias entre las actitudes de los estudiantes hacia los alumnos con discapacidad en función del curso en el que se encuentran los alumnos participantes del estudio.

2.1. Así, esperamos que en los cursos superiores de las titulaciones relacionadas con el ámbito de la educación e integración haya una actitud más favorable que en periodos formativos más tempranos. Con respecto a esto, una de las variables sociodemográficas que lógicamente se relaciona con el curso es la edad de los estudiantes

2.2. Esperamos que, una vez controlada esta covariable, se observen actitudes más positivas conforme avanza la etapa formativa.

\section{Método}

\subsection{Participantes}

La muestra está compuesta por 340 alumnos procedentes de la Universidad de Alicante. De ellos, el $77.65 \%$ fueron mujeres y el $21.18 \%$ eran varones. Dichos alumnos fueron clasificados en distintos grupos en función del curso (110 alumnos pertenecían a $1 .^{\circ}$ de carrera, 121 alumnos a $2 .^{\circ}$ curso y los 108 restantes cursaban $3 .^{\circ}$ ). Asimismo, la muestra de estudiantes estaba distribuida en las titulaciones de Arquitectura $(n=68)$, Informática $(n=43)$, Magisterio $(n=84)$, Biología $(n=53)$, Sociología $(n=42)$ y Trabajo Social $(n=50)$, (Tabla 1$)$.

Tabla 1: Perfil sociodemográfico de los estudiantes

\begin{tabular}{|c|c|c|c|c|c|}
\hline SEXO & N. ${ }^{\circ}$ & Porcentaje & Curso & & \\
\hline Mujer & 264 & 77.65 & 1. ${ }^{\circ}$ Universidad & 111 & 32,65 \\
\hline Varón & 72 & 21.18 & 2. ${ }^{\circ}$ Universidad & 121 & 35,59 \\
\hline No contesta & 4 & 1.18 & 3. ${ }^{\circ}$ Universidad & 108 & 31.76 \\
\hline Total & 340 & 100 & Total & 340 & 100 \\
\hline EDAD & & & TITULACIÓN & & \\
\hline $18-20$ & 40 & 11.76 & Arquitectura & 68 & 20 \\
\hline $21-23$ & 183 & 53.82 & Informática & 43 & 12.65 \\
\hline $24-26$ & 70 & 20.59 & Magisterio & 84 & 24.71 \\
\hline $27-29$ & 18 & 5.29 & Biología & 53 & 15.59 \\
\hline+30 & 13 & 3.82 & Sociología & 42 & 12.35 \\
\hline No contesta & 16 & 4.71 & Trabajo social & 50 & 14.71 \\
\hline Total & 340 & 100 & Total & 340 & 100 \\
\hline
\end{tabular}




\subsection{Instrumentos}

El instrumento empleado ha sido un cuestionario elaborado y validado para el estudio (ver anexo), el cual está formado por dos bloques. En el primero aparecen los datos referentes al alumno, como son, el sexo, el curso, la edad y la titulación.

La segunda parte del instrumento consta de una escala tipo Likert del 1 al 6 ( 1 = Totalmente en desacuerdo, $6=$ Totalmente de acuerdo $)$ de 8 ítems relacionados con las creencias y actitudes de los estudiantes hacia los compañeros con discapacidad.

\subsection{Procedimiento}

Nuestra investigación se basa en un diseño cuasi-experimental, ya que se controlaron algunas variables, como son el género de los alumnos al examinar los grupos en función de las titulaciones, y la edad, al comparar los grupos según el curso. Se eligieron esas titulaciones por ser más fácil acceder a la muestra de estudio. Por otro lado, no se distribuye la población al azar, puesto que los estudiantes pertenecen a contextos concretos y han sido agrupados en función del curso y de la titulación.

\section{Resultados}

Según los resultados indicados por los 340 alumnos de la muestra (Figura 1), observamos que en todos los ítems las puntuaciones medias se encuentran entre estar 1) «totalmente en desacuerdo», en 2) «bastante en desacuerdo»y en 3) «dudo, pero algo en desacuerdo».

Figura 1: Medidas en función de los ítems de la escala

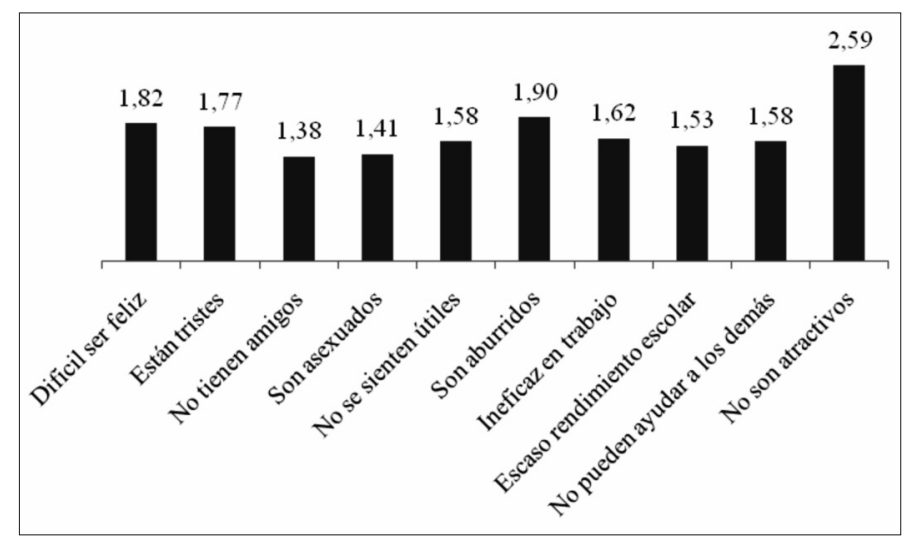

Alternativas, 18, 2011, pp. 75-90 - ISSN 1133-0473 


\section{Dimensiones en función de la titulación}

Para comparar las puntuaciones en función de la titulación se llevó a cabo un análisis multivariante de covarianza (MANCOVA) comparando las diferencias entre los alumnos de las diferentes titulaciones (Biología, Informática, Arquitectura, Trabajo Social, Magisterio y Sociología) y como covariante la variable sexo. Después se realizaron ANCOVA univariantes.

\section{Tabla 2: Puntuaciones medias de cada ítem de la escala} según la titulación y el género

\begin{tabular}{|c|c|c|c|c|c|c|c|c|c|c|}
\hline Ítems & $\begin{array}{l}\text { Item } 1 \\
\text { (No } \\
\text { Feliz) }\end{array}$ & $\begin{array}{l}\text { Ítem } 2 \\
\text { (Triste) }\end{array}$ & $\begin{array}{l}\text { Ítem } 3 \\
\text { (No } \\
\text { Amigos) }\end{array}$ & $\begin{array}{l}\text { Ítem } 4 \\
\text { (No } \\
\text { vida } \\
\text { Sexual) }\end{array}$ & $\begin{array}{l}\text { Ítem } 5 \\
\text { (No útil) }\end{array}$ & $\begin{array}{l}\text { Ítem } 6 \\
\text { (Aburrida) }\end{array}$ & $\begin{array}{l}\text { Ítem } 7 \\
\text { (No } \\
\text { eficaz } \\
\text { Trabajo) }\end{array}$ & $\begin{array}{l}\text { Ítem } 8 \\
\text { (No } \\
\text { eficaz } \\
\text { Escolar) }\end{array}$ & $\begin{array}{l}\text { Item } 9 \\
\text { (No } \\
\text { Ayudar) }\end{array}$ & $\begin{array}{l}\text { Ítem } 10 \\
\text { (No } \\
\text { Atractiva) }\end{array}$ \\
\hline Titulación & $\begin{array}{l}\mathrm{M} \\
\mathrm{D} . \mathrm{T} .\end{array}$ & $\begin{array}{l}\text { M } \\
\text { D.T. }\end{array}$ & $\begin{array}{l}\mathrm{M} \\
\text { D.T. }\end{array}$ & $\begin{array}{l}\text { M } \\
\text { D.T. }\end{array}$ & $\begin{array}{l}\text { M } \\
\text { D.T. }\end{array}$ & $\begin{array}{l}\mathrm{M} \\
\text { D.T. }\end{array}$ & $\begin{array}{l}\text { M } \\
\text { D.T. }\end{array}$ & $\begin{array}{l}\text { M } \\
\text { D.T. }\end{array}$ & $\begin{array}{l}\text { M } \\
\text { D.T. }\end{array}$ & $\begin{array}{l}\text { M } \\
\text { D.T. }\end{array}$ \\
\hline Arquitectura & $\begin{array}{l}1,88 \mathrm{ab} \\
1,07\end{array}$ & $\begin{array}{l}1,65 \mathrm{a} \\
0,72\end{array}$ & $\begin{array}{l}1,61 \text { abed } \\
0,81\end{array}$ & $\begin{array}{l}1,43 \\
0,73\end{array}$ & $\begin{array}{l}2,26 \text { abed } \\
1,57\end{array}$ & $\begin{array}{l}2,61 \text { abed } \\
1,55\end{array}$ & $\begin{array}{l}2,38 \mathrm{abcd} \\
1,33\end{array}$ & $\begin{array}{l}2,00 \mathrm{abc} \\
1,28\end{array}$ & $\begin{array}{l}2,25 \text { abed } \\
1,34\end{array}$ & $\begin{array}{l}2,97 \mathrm{abg} \\
1,52\end{array}$ \\
\hline Informática & $\begin{array}{l}1,65 \mathrm{c} \\
0,72\end{array}$ & $\begin{array}{l}1,58 b \\
0,50\end{array}$ & $\begin{array}{l}1,35 \mathrm{e} \\
0,48\end{array}$ & $\begin{array}{l}1,35 \\
0,69\end{array}$ & $\begin{array}{l}1,67 \mathrm{ae} \\
0,64\end{array}$ & $\begin{array}{l}1,93 \mathrm{ae} \\
1,08\end{array}$ & $\begin{array}{l}1,30 \mathrm{ae} \\
0,46\end{array}$ & $\begin{array}{l}1,63 \\
0,95\end{array}$ & $\begin{array}{l}1,23 \mathrm{ae} \\
0,53\end{array}$ & $\begin{array}{l}2,47 \mathrm{c} \\
0,98\end{array}$ \\
\hline Biología & $\begin{array}{l}2,89 \text { acdef } \\
1,82\end{array}$ & $\begin{array}{l}4,06 \mathrm{abcd} \\
\mathrm{e} \\
6,40\end{array}$ & $\begin{array}{l}2,00 \mathrm{aefg} \\
\mathrm{h} \\
0,96\end{array}$ & $\begin{array}{l}1,81 \mathrm{ab} \\
1,19\end{array}$ & $\begin{array}{l}1,89 \mathrm{fgh} \\
1,03\end{array}$ & $\begin{array}{l}2,64 \text { efgh } \\
1,47\end{array}$ & $\begin{array}{l}2,40 \text { efgh } \\
1,18\end{array}$ & $\begin{array}{l}2,04 \text { def } \\
1,24\end{array}$ & $\begin{array}{l}2,06 \text { efgh } \\
0,86\end{array}$ & $\begin{array}{l}3,64 \mathrm{cdef} \\
1,48\end{array}$ \\
\hline $\begin{array}{l}\text { Sociologia } \\
\text { Trabajo } \\
\text { Social }\end{array}$ & $\begin{array}{l}1,62 \mathrm{~d} \\
1,03 \\
1,20 \mathrm{be} \\
0,75\end{array}$ & $\begin{array}{l}1,25 \mathrm{c} \\
0,52 \\
1,14 \mathrm{~d} \\
0,44\end{array}$ & $\begin{array}{l}1,08 \mathrm{bf} \\
0,33 \\
1,20 \mathrm{cg} \\
0,64\end{array}$ & $\begin{array}{l}1,21 \mathrm{a} \\
0,57 \\
1,21 \mathrm{~b} \\
0,87\end{array}$ & $\begin{array}{l}1,10 \mathrm{bef} \\
0,41 \\
1,13 \mathrm{cg} \\
0,38\end{array}$ & $\begin{array}{l}1,46 \mathrm{bf} \\
0,54 \\
1,38 \mathrm{cg} \\
0,62\end{array}$ & $\begin{array}{l}1,13 \mathrm{bf} \\
0,60 \\
1,13 \mathrm{cg} \\
0,51\end{array}$ & $\begin{array}{l}1,12 \mathrm{ad} \\
0,51 \\
1,18 \text { be } \\
0,64\end{array}$ & $\begin{array}{l}1,13 \mathrm{bf} \\
0,53 \\
1,14 \mathrm{cg} \\
0,44\end{array}$ & $\begin{array}{l}2,08 \mathrm{adg} \\
1,13 \\
1,77 \mathrm{be} \\
1,13\end{array}$ \\
\hline Magisterio & $\begin{array}{l}1,73 \mathrm{f} \\
1,37\end{array}$ & $\begin{array}{l}1,27 \mathrm{e} \\
0,84\end{array}$ & $\begin{array}{l}1,13 \mathrm{dh} \\
0,46\end{array}$ & $\begin{array}{l}1,44 \\
0,86 \\
\end{array}$ & $\begin{array}{l}1,35 \mathrm{dh} \\
0,86\end{array}$ & $\begin{array}{l}1,44 \mathrm{dh} \\
0,63\end{array}$ & $\begin{array}{l}1,29 \mathrm{dh} \\
0,77\end{array}$ & $\begin{array}{l}1,24 \mathrm{cf} \\
0,67\end{array}$ & $\begin{array}{l}1,45 \mathrm{dh} \\
1,06\end{array}$ & $\begin{array}{l}2,51 \mathrm{f} \\
1,46\end{array}$ \\
\hline F & $11.57 * *$ & $10.55 * *$ & $17.50 * *$ & $3.61 * *$ & $14.92 * *$ & $19.97^{* *}$ & $31.41^{* *}$ & $13.50^{* *}$ & $18.84 * *$ & $14.55 * *$ \\
\hline Sexo & $\begin{array}{l}\text { M } \\
\text { D.T. }\end{array}$ & $\begin{array}{l}\text { M } \\
\text { D.T. }\end{array}$ & $\begin{array}{l}\text { M } \\
\text { D.T. }\end{array}$ & $\begin{array}{l}\text { M } \\
\text { D.T. }\end{array}$ & $\begin{array}{l}\text { M } \\
\text { D.T. }\end{array}$ & $\begin{array}{l}\text { M } \\
\text { D.T. }\end{array}$ & $\begin{array}{l}\text { M } \\
\text { D.T. }\end{array}$ & $\begin{array}{l}\text { M } \\
\text { D.T. }\end{array}$ & $\begin{array}{l}\text { M } \\
\text { D.T. }\end{array}$ & $\begin{array}{l}\text { M } \\
\text { D.T. }\end{array}$ \\
\hline Varón & $\begin{array}{l}1,83 \\
1,39\end{array}$ & $\begin{array}{l}2,51 \\
4,52\end{array}$ & $\begin{array}{l}1,38 \\
0,68\end{array}$ & $\begin{array}{l}1,24 \\
0,66\end{array}$ & $\begin{array}{l}1,74 \\
1,16\end{array}$ & $\begin{array}{l}2,03 \\
1,27\end{array}$ & $\begin{array}{l}1,61 \\
1,00\end{array}$ & $\begin{array}{l}1,58 \\
0,95\end{array}$ & $\begin{array}{l}1,57 \\
1,02\end{array}$ & $\begin{array}{l}2,43 \\
1,40\end{array}$ \\
\hline Mujer & $\begin{array}{l}1,83 \\
1,28 \\
\end{array}$ & $\begin{array}{l}1,59 \\
1,96 \\
\end{array}$ & $\begin{array}{l}1,38 \\
0,73 \\
\end{array}$ & $\begin{array}{l}1,46 \\
0,89 \\
\end{array}$ & $\begin{array}{l}1,54 \\
1,02 \\
\end{array}$ & $\begin{array}{l}1,87 \\
1,17 \\
\end{array}$ & $\begin{array}{l}1,63 \\
1,07 \\
\end{array}$ & $\begin{array}{l}1,51 \\
1,01 \\
\end{array}$ & $\begin{array}{l}1,58 \\
1,01 \\
\end{array}$ & $\begin{array}{l}2,63 \\
1,46 \\
\end{array}$ \\
\hline $\mathrm{T}$ & 0.03 & $7.35 *$ & 2.39 & $3.97 *$ & 0.27 & 1.37 & $8.02 *$ & 1.42 & $4.01 \%$ & 3.66 \\
\hline
\end{tabular}

(aa, bb...) Pares de letras iguales en cada columna indican diferencias estadísticamente significativas entre las medias de los grupos en cada ítem; $\left({ }^{*}\right)=$ Nivel de significación de $.001 ;\left(^{*}\right)=$ Nivel de significación de .05 .

En la Tabla 2 se presenta el MANCOVA, los ANOVAS y los valores medios obtenidos para cada uno de los ítems de la escala.

El MANCOVA general fue significativo (Lambda de Wilks $10,340=40.513$, $\mathrm{p}=.000)$.

Al examinar las pruebas post hoc para averiguar entre qué grupos se encontraron las diferencias una vez controlada la covariable sexo observamos que la mayoría de índices centrales más bajos están distribuidos entre los alumnos de titulaciones que guardan relación con la atención a la diversidad, es decir, con Sociología, Magisterio y Trabajo Social, al compararlos con las medias de los otros grupos de titulaciones como Biología y Arquitectura, los alumnos 
de estas carreras muestran actitudes menos positivas que los alumnos de Sociología, Magisterio y Trabajo Social.

Con referencia a la covariante sexo observamos que su influencia afecta a algunos ítems como el que hace referencia a que «Normalmente las personas con discapacidad son y/o están tristes», destacó el grupo de los varones con una media superior $(\mathrm{M}=2.51, \mathrm{DT}=4.52)$ a la de las mujeres $(\mathrm{M}=1.59, \mathrm{DT}=1.96)$, en la afirmación relativa a «Las personas con discapacidad son inocentes y no deben tener actividad sexual» en la que encontramos una media de 1.57 (DT $=0.98)$ en el grupo de los varones con respecto al grupo de mujeres $(\mathrm{M}=1.26$, DT $=0.77$ ), al ítem «Una persona con discapacidad será poco eficaz en su trabajo», observándose que los varones están más de cuerdo con esta afirmación que las mujeres $(\mathrm{M}=2.02, \mathrm{DT}=1.30)$, y finalmente en el ítem relativo a que «Una persona con discapacidad será poco eficaz en su trabajo», destaca el grupo de varones con una media superior $(\mathrm{M}=2.02, \mathrm{DT}=1.30)$ respecto al de mujeres.

\section{Dimensiones en función del curso}

Para comparar las puntuaciones en función del curso se llevó a cabo un análisis multivariante de covarianza (MANCOVA) comparando las diferencias entre los alumnos de los diferentes cursos $\left(1 .^{\circ}, 2 .^{\circ}\right.$ y $\left.3 .^{\circ}\right)$ y como covariante la variable edad. En la tabla 3 se presentan los resultados de los ANCOVA univariantes.

Como puede verse (Tabla 3), el grupo de estudiantes de primer curso presenta las puntuaciones más altas de los cursos analizados, es decir, los que más de acuerdo están con estas afirmaciones.

$\mathrm{Al}$ analizar las dimensiones de la escala en función del curso observamos que existen diferencias en todos los ítems según el curso en el que se encuentren los alumnos. Así, a nivel descriptivo, vemos que ante el ítem referente a «Es muy difícil que las personas con discapacidad sean felices», la media de los alumnos que cursan $1 .^{\circ}(\mathrm{M}=2.30, \mathrm{DT}=1.59)$ superan con una media más alta a los alumnos que cursan el resto de cursos.

Ante la afirmación relativa a creer que «Normalmente las personas con discapacidad son y/o están tristes», encontramos que la media de los alumnos de $1 .^{\circ}(\mathrm{M}=2.64, \mathrm{DT}=4.58)$ alcanzaron puntuaciones medias más altas que los estudiantes de $2 .^{\circ}$ y $3 .^{\circ}$.

Con respecto al ítem que hace referencia a que «Una persona con discapacidad tendrá pocos amigos/as», observamos que el curso de $1 .^{\circ}(\mathrm{M}=1.59, \mathrm{DT}=0.87)$ se diferencia en su índice central a la media de los alumnos de $2 .^{\circ}(\mathrm{M}=1.09$, DT $=0.43$ ). A su vez, observamos que la media de este último curso $\left(2 .^{\circ}\right)$ es estadisticamente inferior a la de los estudiantes de $3 .^{\circ}(\mathrm{M}=1.38, \mathrm{DT}=0.75)$. 
Tabla 3: Puntuaciones medias de cada ítem de la escala según el curso y edad

\begin{tabular}{|c|c|c|c|c|c|c|c|c|c|c|}
\hline Ítems & $\begin{array}{l}\text { Ítem } 1 \\
\text { (No } \\
\text { Feliz) }\end{array}$ & $\begin{array}{l}\text { Item } 2 \\
\text { (Triste) }\end{array}$ & $\begin{array}{l}\text { Ítem } 3 \\
\text { (No } \\
\text { Amigos) }\end{array}$ & $\begin{array}{l}\text { Ítem } 4 \\
\text { (No vida } \\
\text { Sexual) }\end{array}$ & $\begin{array}{l}\text { Ítem } 5 \\
\text { (No útil) }\end{array}$ & $\begin{array}{l}\text { Item } 6 \\
\text { (Aburrida) }\end{array}$ & $\begin{array}{l}\text { Item } 7 \\
\text { (No } \\
\text { eficaz } \\
\text { Trabajo) }\end{array}$ & $\begin{array}{l}\text { Ítem } 8 \\
\text { (No } \\
\text { eficaz } \\
\text { Escolar) }\end{array}$ & $\begin{array}{l}\text { Ítem } 9 \\
\text { (No } \\
\text { Ayudar) }\end{array}$ & $\begin{array}{l}\text { Ítem } 10 \\
\text { (No } \\
\text { Atractiva) }\end{array}$ \\
\hline Curso & $\begin{array}{l}\text { M } \\
\text { D.T. }\end{array}$ & $\begin{array}{l}\text { M } \\
\text { D.T. }\end{array}$ & $\begin{array}{l}\text { M } \\
\text { D.T. }\end{array}$ & $\begin{array}{l}\text { M } \\
\text { D.T. }\end{array}$ & $\begin{array}{l}\text { M } \\
\text { D.T. }\end{array}$ & $\begin{array}{l}\mathrm{M} \\
\text { D.T. }\end{array}$ & $\begin{array}{l}\text { M } \\
\text { D.T. }\end{array}$ & $\begin{array}{l}\text { M } \\
\text { D.T. }\end{array}$ & $\begin{array}{l}\text { M } \\
\text { D.T. }\end{array}$ & $\begin{array}{l}\text { M } \\
\text { D.T. }\end{array}$ \\
\hline $1^{\circ}$ & $\begin{array}{l}2,30 \mathrm{ab} \\
1,59\end{array}$ & $\begin{array}{l}2,64 \mathrm{ab} \\
4,58\end{array}$ & $\begin{array}{l}1,59 b \\
0,87\end{array}$ & $\begin{array}{l}1,57 \mathrm{a} \\
0,98\end{array}$ & $\begin{array}{l}1,84 \mathrm{ab} \\
1,39\end{array}$ & $\begin{array}{l}2,41 \text { bc } \\
1,49\end{array}$ & $\begin{array}{l}2,02 \mathrm{bc} \\
1,30\end{array}$ & $\begin{array}{l}1,86 \mathrm{ab} \\
1,29\end{array}$ & $\begin{array}{l}1,96 \mathrm{ab} \\
1,25\end{array}$ & $\begin{array}{l}3,01 \mathrm{ab} \\
1,55\end{array}$ \\
\hline 2 & $\begin{array}{l}1,49 b \\
1,19\end{array}$ & $\begin{array}{l}1,20 b \\
0,72\end{array}$ & $\begin{array}{l}1,09 \mathrm{ab} \\
0,43\end{array}$ & $\begin{array}{l}1,31 \\
0,77\end{array}$ & $\begin{array}{l}1,24 b \\
0,72\end{array}$ & $\begin{array}{l}1,40 \mathrm{ac} \\
0,59\end{array}$ & $\begin{array}{l}1,20 \mathrm{ac} \\
0,65\end{array}$ & $\begin{array}{l}1,18 b \\
0,58\end{array}$ & $\begin{array}{l}1,27 b \\
0,81\end{array}$ & $\begin{array}{l}2,14 b \\
1,31\end{array}$ \\
\hline 3 & $\begin{array}{l}1,46 a \\
0,85\end{array}$ & $\begin{array}{l}1,38 \mathrm{a} \\
0,67\end{array}$ & $\begin{array}{l}1,38 \mathrm{a} \\
0,75\end{array}$ & $\begin{array}{l}1,26 a \\
0,77\end{array}$ & $\begin{array}{l}1,46 a \\
0,85\end{array}$ & $\begin{array}{l}1,76 \mathrm{ab} \\
1,05\end{array}$ & $\begin{array}{l}1,60 \mathrm{ab} \\
1,02\end{array}$ & $\begin{array}{l}1,38 \mathrm{a} \\
0,77\end{array}$ & $\begin{array}{l}1,44 a \\
0,72\end{array}$ & $\begin{array}{l}2,35 \mathrm{a} \\
1,43\end{array}$ \\
\hline$T$ & $\begin{array}{l}1,74 \\
1,30 \\
\end{array}$ & $\begin{array}{l}1,73 \\
2,75 \\
\end{array}$ & $\begin{array}{l}1,35 \\
0,73 \\
\end{array}$ & $\begin{array}{l}1,38 \\
0,85\end{array}$ & $\begin{array}{l}1,51 \\
1,05 \\
\end{array}$ & $\begin{array}{l}1,85 \\
1,17 \\
\end{array}$ & $\begin{array}{l}1,60 \\
1,07 \\
\end{array}$ & $\begin{array}{l}1,47 \\
0,97\end{array}$ & $\begin{array}{l}1,55 \\
0,99\end{array}$ & $\begin{array}{l}2,49 \\
1,47 \\
\end{array}$ \\
\hline$F$ & 15,054 & 8,295 & 13,024 & $4,016^{*}$ & 9,133 & 22,653 & 17,534 & 14,097 & 14,138 & 11,434 \\
\hline Edad & $\begin{array}{l}\text { M } \\
\text { D.T. }\end{array}$ & $\begin{array}{l}\text { M } \\
\text { D.T. }\end{array}$ & $\begin{array}{l}\text { M } \\
\text { D.T. }\end{array}$ & $\begin{array}{l}\text { M } \\
\text { D.T. }\end{array}$ & $\begin{array}{l}\text { M } \\
\text { D.T. }\end{array}$ & $\begin{array}{l}\text { M } \\
\text { D.T. }\end{array}$ & $\begin{array}{l}\text { M } \\
\text { D.T. }\end{array}$ & $\begin{array}{l}\text { M } \\
\text { D.T. }\end{array}$ & $\begin{array}{l}\text { M } \\
\text { D.T. }\end{array}$ & $\begin{array}{l}\text { M } \\
\text { D.T. }\end{array}$ \\
\hline $18-20$ & $\begin{array}{l}2,01 \\
1,65\end{array}$ & $\begin{array}{l}3,09 \mathrm{ab} \\
5,75\end{array}$ & $\begin{array}{l}1,49 \\
0,83\end{array}$ & $\begin{array}{l}1,43 \\
0,99\end{array}$ & $\begin{array}{l}1,73 \\
1,25\end{array}$ & $\begin{array}{l}2,14 a \\
1,38\end{array}$ & $\begin{array}{l}1,69 \\
1,11\end{array}$ & $\begin{array}{l}1,67 \\
1,11\end{array}$ & $\begin{array}{l}1,83 \mathrm{a} \\
1,26\end{array}$ & $\begin{array}{l}2,70 \\
1,51\end{array}$ \\
\hline $21-23$ & $\begin{array}{l}1,80 \\
1,15\end{array}$ & $\begin{array}{l}1,46 b \\
0,74\end{array}$ & $\begin{array}{l}1,40 \\
0,73\end{array}$ & $\begin{array}{l}1,45 \\
0,83\end{array}$ & $\begin{array}{l}1,64 \\
1,07\end{array}$ & $\begin{array}{l}1,96 \\
1,22\end{array}$ & $\begin{array}{l}1,76 a \\
1,14\end{array}$ & $\begin{array}{l}1,52 \\
1,00\end{array}$ & $\begin{array}{l}1,61 \\
0,94\end{array}$ & $\begin{array}{l}2,66 \\
1,48\end{array}$ \\
\hline $24-26$ & $\begin{array}{l}1,57 \\
1,33\end{array}$ & $\begin{array}{l}1,31 \mathrm{a} \\
0,98\end{array}$ & $\begin{array}{l}1,24 \\
0,48\end{array}$ & $\begin{array}{l}1,33 \\
1,03\end{array}$ & $\begin{array}{l}1,21 \\
0,72\end{array}$ & $\begin{array}{l}1,45 \mathrm{a} \\
0,50\end{array}$ & $\begin{array}{l}1,19 a \\
0,77\end{array}$ & $\begin{array}{l}1,24 \\
0,73\end{array}$ & $\begin{array}{l}1,19 \mathrm{a} \\
0,59\end{array}$ & $\begin{array}{l}2,48 \\
1,37\end{array}$ \\
\hline $27-29$ & $\begin{array}{l}1,68 \\
0,99\end{array}$ & $\begin{array}{l}1,77 \\
0,61\end{array}$ & $\begin{array}{l}1,23 \\
0,61\end{array}$ & $\begin{array}{l}1,32 \\
0,48\end{array}$ & $\begin{array}{l}1,68 \\
0,72\end{array}$ & $\begin{array}{l}2,18 \\
1,05\end{array}$ & $\begin{array}{l}1,59 \\
0,80\end{array}$ & $\begin{array}{l}1,73 \\
0,98\end{array}$ & $\begin{array}{l}1,32 \\
0,95\end{array}$ & $\begin{array}{l}2,09 \\
0,97\end{array}$ \\
\hline+30 & $\begin{array}{l}1,94 \\
1,43\end{array}$ & $\begin{array}{l}1,53 \\
0,62\end{array}$ & $\begin{array}{l}1,29 \\
0,85\end{array}$ & $\begin{array}{l}1,18 \\
0,53\end{array}$ & $\begin{array}{l}1,24 \\
0,97\end{array}$ & $\begin{array}{l}1,41 \\
1,23\end{array}$ & $\begin{array}{l}1,06 \\
0,24\end{array}$ & $\begin{array}{l}1,71 \\
1,21 \\
\end{array}$ & $\begin{array}{l}1,29 \\
0,99\end{array}$ & $\begin{array}{l}1,94 \\
1,09\end{array}$ \\
\hline$F$ & 0,887 & $5,191 * *$ & 1,107 &, 570 & 2,276 & $3,355^{*}$ & $3,922^{*}$ & 1,573 & $3,568^{*}$ & 1,828 \\
\hline
\end{tabular}

(aa, bb...) Pares de letras iguales en cada columna indican diferencias estadísticamente significativas entre las medias de los grupos en cada ítem; $(* *)=$ Nivel de significación de $.001 ;\left(^{*}\right)=$ Nivel de significación de .05 .

En la afirmación relativa a «Las personas con discapacidad son inocentes y no deben tener actividad sexual» encontramos una media de $1.57(\mathrm{DT}=0.98)$ en $1 .{ }^{\circ}$, observándose diferencias significativas con el curso de $3 .^{\circ}(\mathrm{M}=1.26$, DT $=0.77$ ).

Con respecto a la creencia de que «Una persona con discapacidad difícilmente podrá sentirse útil», observamos que el grupo de $1 .^{\circ}(\mathrm{M}=1.84$, DT $=1.39)$ destacó con una media significativamente mayor que las del resto de cursos.

Ante la afirmación relativa a creer que «Una persona con discapacidad tendrá una vida aburrida», encontramos que los alumnos con puntuaciones medias más altas fueron los alumnos de $1 .^{\circ}(\mathrm{M}=2.41$, DT $=1.49)$, observándose un resultado significativo con los otros grupos. Asimismo, se observaron diferencias entre $2 .^{\circ}(\mathrm{M}=1.40, \mathrm{DT}=0.59)$ y $3 .^{\circ}(\mathrm{M}=1.76, \mathrm{DT}=1.05)$.

En cuanto al ítem «Una persona con discapacidad será poco eficaz en su trabajo», observamos que el curso de $1 .^{\circ}$ destacó con una media superior (M $=2.02, \mathrm{DT}=1.30$ ) respecto a $2 .^{\circ}$ y $3 .^{\circ}$. A su vez, se observaron diferencias entre $2 .^{\circ}(\mathrm{M}=1.20, \mathrm{DT}=0.65)$ y $3 .^{\circ}(\mathrm{M}=1.60, \mathrm{DT}=1.02)$. 
Con respecto a «Una persona con discapacidad tendrá escaso rendimiento escolar», observamos que, de nuevo, el curso de $1 .{ }^{\circ}(\mathrm{M}=1.86, \mathrm{DT}=1.29)$ resultó significativo al compararlo con el resto.

Con referencia a creer que «Es difícil que una persona con discapacidad pueda ayudar a los demás», observamos puntuaciones medias más altas en $1 .^{\circ}$ $(\mathrm{M}=1.96, \mathrm{DT}=1.25)$, en comparación con los otros cursos.

Finalmente, observamos los mismos resultados en la opinión referente a que «Las personas con discapacidad no resultan atractivas para otras personas», es decir, encontramos que los alumnos que cursan $1 .{ }^{\circ}$ destacaron con una media más alta $(\mathrm{M}=3.01, \mathrm{DT}=1.55)$ que el alumnado del resto de cursos.

Con respecto a la covariable edad, observamos que solo influye en algunos ítems. Así, encontramos que los estudiantes entre 18 y 20 años de edad son los que están más de acuerdo en afirmar que «es muy difícil que las personas con discapacidad sean felices». Asimismo, este rango de edad destacó en el ítem que hace referencia a que «una persona con discapacidad tendrá una vida aburrida», en la creencia de que «una persona con discapacidad será poco eficaz en su trabajo» y en el ítem referente a que «es difícil que una persona discapacitada pueda ayudar a los demás».

\section{Discusión}

La importancia de este trabajo parte de la necesidad de evaluar las actitudes de los universitarios hacia los estudiantes con discapacidad, ya que estas son consideradas el primer elemento que puede facilitar o dificultar el proceso de integración e inclusión de alumnos con necesidades educativas especiales en educación superior (Reina, 2003; Santos Rego y Lorenzo Moledo, 2010). Así, en general, nuestros resultados indican que los alumnos muestran actitudes favorables hacia los alumnos con discapacidad. Ninguna de las puntuaciones medias indica estar de acuerdo con actitudes desfavorables.

$\mathrm{Si}$ indagamos en las dimensiones en las que se observa mayor desacuerdo podemos destacar las referentes a que «Las personas con discapacidad son inocentes y no deben tener actividad sexual», «Tendrán pocos amigos» y «Tendrán escaso rendimiento», mientras que existe mayor acuerdo o duda en que «Las personas con discapacidad no resultan atractivas para otras personas» y que «Una persona con discapacidad tendrá una vida aburrida».

En cuanto a las actitudes en función de la titulación de los estudiantes universitarios, se pone de manifiesto que en todos los casos hay una buena aceptación de la integración del alumnado con discapacidad en la universidad, independientemente de la carrera o titulación en la que los estudiantes están matriculados. Sin embargo, hemos de mencionar que existe un nivel compara- 
tivamente más desfavorable hacia la discapacidad entre los estudiantes de las titulaciones de Arquitectura y de Biología; mientras que es comparativamente más positivo en la mayoría de ítems entre los alumnos de Trabajo Social, Sociología y Magisterio. De este modo, podemos afirmar que se cumple nuestra primera hipótesis, puesto que nos encontramos con que los alumnos que estudian carreras más relacionadas con el trato o atención hacia la discapacidad muestran unas actitudes más favorables.

Estos resultados están en línea con los obtenidos por otros autores (Alonso, Navarro y Vicente, 2008; Santos Rego y Lorenzo Moledo, 2010), los cuales examinaron las actitudes hacia la diversidad mostradas por los estudiantes universitarios de diversas áreas de conocimiento, encontrando que en los alumnos que cursaban carreras referentes a Humanidades y Educación son más positivas que en los estudiantes universitarios de otras áreas de conocimiento como las titulaciones Técnicas y de Ciencias Experimentales.

Al analizar el posible efecto del sexo en las actitudes en función de la titulación hemos visto que, tras controlar su influencia, siguen existiendo diferencias a nivel significativo entre las titulaciones, encontrando que influye en algunos ítems de la escala, siendo los varones los que muestran una actitud menos favorable hacia las personas con discapacidad.

Nuestros resultados van en la misma dirección que otros estudios (Olkin y Howson, 1994; Stovall y Sedlacek, 1983) en los que encontraron que las mujeres mostraron actitudes más positivas hacia las personas con discapacidad que los hombres.

En cuanto a la hipótesis planteada concerniente a las diferentes actitudes en función del curso de los estudiantes, observamos que el nivel cursado en el que los alumnos son más jóvenes muestra actitudes menos favorables que en los cursos superiores. En este sentido, podríamos pensar que la evolución de las actitudes guardan relación con la variable edad, sin embargo, al controlar la covariante edad observamos que los resultados continúan siendo estadísticamente significativos, indicando solo en algunos ítems de la escala la influencia de la edad. Por tanto, parece que la evolución de unas actitudes más favorables aumenta no tanto conforme los estudiantes crecen sino con su progreso en su formación a través de los diferentes cursos (Aguado, Flórez y Alcedo, 2004).

Estos resultados siguen la misma línea que los datos obtenidos en la investigación de Infante y Gómez (2004) en los que se destacó un aumento hacia actitudes positivas en el último curso si se compara con el primero de estudiantes de educación superior.

Por tanto, la relevancia de esta investigación en el ámbito educativo es clara porque las actitudes se consideran una de las variables más importantes para la integración de los alumnos con discapacidad. 
Ello sugiere la importancia de evaluar las actitudes de los estudiantes y, con ello, la necesidad, todavía existente, de implantar programas de sensibilización que potencien unas actitudes favorables desde los primeros cursos e independientemente del área de conocimiento por la que se incline un estudiante.

No obstante, aunque se cumplen las hipótesis del estudio, consideramos que existen elementos que pueden mejorar la investigación, como por ejemplo:

- Sería interesante realizar un postest con los estudiantes que han participado en esta investigación una vez se encuentren en activo para comprobar si se mantienen los resultados obtenidos cuando han tenido la posibilidad de poner en práctica los conocimientos adquiridos.

- Por otro lado, en posteriores investigaciones, sería de interés considerar la posibilidad de valorar otras titulaciones, así como las actitudes mostradas por los estudiantes de otras universidades, con el fin de comparar los datos obtenidos y comprobar si pueden generalizarse los resultados de la investigación a otra población.

- Finalmente, y considerando que las actitudes van evolucionando positivamente conforme avanzan los cursos, se podría plantear la propuesta de incluir programas con contenidos que hagan referencia a la sensibilización e integración de las personas con discapacidad al inicio de la etapa universitaria en todas las titulaciones.

\section{Referencias bibliográficas}

Aguado, A. L., Flórez, M. A. y Alcedo, M. A. (2004). Programas de cambio de actitudes ante la discapacidad. Psicothema, 16, 667-673.

Alpuín, G., González, M. A. y Pérez, M. (2006, mayo). Creencias y actitudes de los estudiantes del último curso de magisterio hacia la atención a la diversidad. Comunicación presentada en las XI Jornadas de Fomento de la Investigación, Universitat Jaume I, Castellón.

Bunch, G., y Valeo, A. (2004). Student attitudes toward peers with disabilities in inclusive and special education schools. Disability \& Society, 19, 61-76.

Burstein, N., Sears, S., Wilcoxen, A., Cabello, B. y Spagna, M. (2004). Moving toward inclusive practices. Remedial and Special Education, 2, 104-116.

Castellana, M. y Sala, I. (2005, noviembre). Estudiantes con discapacidad en las aulas universitarias. Estudio sobre la atención a la diversidad dentro de las aulas universitarias. Trabajo presentado en el I Congreso sobre Universidad y Discapacidad. Salamanca.

Cuskelly, M. y Bryde, R. (2004). Attitudes towards adults with a intellectual disability: parents, support staff and community sample. Journal of Intellectual and Developmental Disability, 19, 255-264.

Eches, S. y Ochoa, T. (2005). Students with disabilities: Transitioning from High School to higher education. American Secondary Education, 33, 6-20. 
Estévez, E., Murgui, S., Moreno, D. y Musitu, G. (2007). Estilos de comunicación familiar, actitud hacia la autoridad institucional y conducta violenta del adolescente en la escuela. Psicothema, 19, 108-113.

Garaigordobil, M. y García de Galdeano, P. (2006). Empatía en niños de 10 a 12 años. Psicothema, 18, 180-186.

Infante, M. y Gómez, V. (2004). Actitudes de los estudiantes de Educación hacia la integración de personas con discapacidad y hacia la educación multicultural. Cultura y Educación, 16, 371-384.

Konur, O. (2006). Teaching disabled students in higher education. Teaching in Higher Education, 11, 351- 363.

Lynch, K. y Feeley, M. (2009). Gender and Education (and Employment): Gendered imperatives and their implications for women and men. Lessons from Research for Policy Makers. European Commission, Brussels. Obtenido el 01 de junio de 2010 en http://www.nesse.fr/nesse/activitie

Moreno J., Rodríguez I., Saldaña D. y Aguilera A. (2006). Actitudes ante la discapacidad en el alumnado universitario matriculado en materias afines. Revista Iberoamericana de Educación, 40, 5-25.

Nowicki, E. A. (2006). A cross-sectional multivariate análisis of children's attitudes towards disabilities. Journal of Intellectual Disability Research, 50, 335-348.

Olkin, R. y Howson, L. J. (1994). Attitudes toward and images of physical disability. Journal of Social Behavior and Personality, 9, 81-96.

Santos Rego, M. A. y Lorenzo Moledo, M. M. (2010). La dimensión cívica en el desarrollo formativo de los estudiantes universitarios. Revista Electrónica de Investigación Educativa. Obtenido el 15 de junio de 2010 en http://redie.uabc.mx/NumEsp2/contenido-rego.html

Stovall, C. y Sedlacek, W. E. (1983). Attitudes of male and female university students toward students with different physical disabilities. Journal of College Student Personnel, 24, 325-330.

Verdugo, M. A. Arias, B. y Jenaro, C. (1994). Actitudes hacia las personas con minusvalía. Madrid: Ministerio de Asuntos Sociales, Instituto Nacional de Servicios Sociales. 



\section{A N EX O}

\section{U E S T I O N A R I O}

Indica tu grado de acuerdo o desacuerdo con cada una de las siguientes afirmaciones, según esta numeración:

- Totalmente de acuerdo: (6)

- Bastante de acuerdo: (5)

- Algo de acuerdo, pero dudo: (4)

- Dudo, pero más bien en desacuerdo: (3)

- Bastante en desacuerdo: (2)

- Totalmente en desacuerdo: (1)

\begin{tabular}{|l|l|l|l|l|l|l|}
\hline \multicolumn{1}{|c|}{ Bloque 1. } \\
Sexo
\end{tabular}




\begin{tabular}{|c|c|c|c|c|c|c|}
\hline Bloque $1 .^{\circ}$ & \multicolumn{6}{|c|}{ Datos sociodemográficos } \\
\hline Sexo & \multicolumn{3}{|l|}{ Varón } & \multicolumn{3}{|l|}{ Mujer } \\
\hline \multicolumn{7}{|l|}{ Edad } \\
\hline Titulación & & & & Curso & & \\
\hline Bloque $2 .^{\circ}$ & \multicolumn{6}{|c|}{ Actitudes hacia la discapacidad } \\
\hline Escala & $\begin{array}{l}\text { Totalmente } \\
\text { de acuerdo }\end{array}$ & $\begin{array}{c}\text { Bastante } \\
\text { de } \\
\text { acuerdo }\end{array}$ & $\begin{array}{c}\text { Algo de } \\
\text { acuerdo, } \\
\text { pero dudo }\end{array}$ & \begin{tabular}{|l|} 
Dudo, pero \\
más bien en \\
desacuerdo
\end{tabular} & $\begin{array}{l}\text { Bastante } \\
\text { en de- } \\
\text { sacuerdo }\end{array}$ & $\begin{array}{c}\text { Totalmente } \\
\text { en } \\
\text { desacuerdo }\end{array}$ \\
\hline $\begin{array}{l}\text { Una persona con } \\
\text { discapacidad tendrá } \\
\text { una vida aburrida. }\end{array}$ & & & & & & \\
\hline $\begin{array}{l}\text { Una persona con } \\
\text { discapacidad será } \\
\text { poco eficaz en su } \\
\text { trabajo. }\end{array}$ & & & & & & \\
\hline $\begin{array}{l}\text { Una persona con } \\
\text { discapacidad tendrá } \\
\text { escaso rendimiento } \\
\text { escolar. }\end{array}$ & & & & & & \\
\hline $\begin{array}{l}\text { Es difícil que una } \\
\text { persona con } \\
\text { discapacidad pueda } \\
\text { ayudar a los demás. }\end{array}$ & & & & & & \\
\hline $\begin{array}{l}\text { Las personas con } \\
\text { discapacidad no } \\
\text { resultan atractivas } \\
\text { para otras personas. }\end{array}$ & & & & & & \\
\hline
\end{tabular}

Muchas gracias 